\title{
Effects of a 1-Day Training Course in Dermoscopy Among General Practitioners
}

\author{
Anna Augustsson ${ }^{1}$, John Paoli ${ }^{2,3}$
}

1 Praktikertjänst AB, Centrumpraktiken Kungälv, Sweden

2 Department of Dermatology and Venereology, Institute of Clinical Sciences at the Sahlgrenska Academy, University of Gothenburg, Sweden 3 Region Västra Götaland, Sahlgrenska University Hospital, Department of Dermatology and Venereology, Gothenburg, Sweden

Key words: dermoscopy, general medicine, diagnostic accuracy, malignant melanoma, nonmelanoma skin cancer

Citation: Augustsson A, Paoli J. Effects of a 1-day training course in dermoscopy among general practitioners. Dermatol Pract Concept. 2019;9(3):195-199. DOI: https://doi.org/10.5826/dpc.0903a04

Accepted: May 20, 2019; Published: July 31, 2019

Copyright: (92019 Augustsson and Paoli. This is an open-access article distributed under the terms of the Creative Commons Attribution License, which permits unrestricted use, distribution, and reproduction in any medium, provided the original author and source are credited.

Funding: None.

Competing interests: The authors have no conflicts of interest to disclose.

Authorship: Both authors have contributed significantly to this publication.

Corresponding author: Anna Augustsson, MD, Resident in General Medicine Praktikertjänst, Centrumpraktiken Kungälv, Triogatan 5, 442 43 Kungälv, Sweden. Email: anna.augustsson@ptj.se

ABSTRACT Background: General practitioners (GPs) are often the first point of contact for Swedish patients seeking medical advice for skin lesions of concern, but many lack training in dermoscopy.

Objective: To examine the effects of a 1-day training course in dermoscopy among Swedish GPs.

Methods: The intervention group consisted of GPs who underwent a 1-day training course in dermoscopy and a control group that did not undergo any education. Before the training course, the intervention group performed a test consisting of 30 dermoscopy cases including 9 different benign and malignant melanocytic and nonmelanocytic diagnoses. The participants then took the same test directly after the course and again after 6 months. The control group took the same test twice with a 6-month interval in between tests in order to avoid recall bias.

Results: Twenty-seven GPs in the intervention group took the test before and immediately after the course with an improvement of their median test scores by 8 points ( 13 vs 20 correct answers, $\mathrm{P}<$ 0.01 ). Eighteen participants also took the test a third time after 6 months with similar results compared with the second test (median scores of 20.5 vs $20.0, P=0.3$ ). In the control group, 16 persons preformed both tests with an improvement of their median score by 2 points ( 13.5 vs 15.5 correct answers, $\mathrm{P}=0.06$ ).

Conclusions: The results of this study show positive effects on diagnostic accuracy in a test situation among GPs receiving a 1-day training course in dermoscopy. 


\section{Introduction}

The incidence of malignant melanoma in Sweden has increased by $5 \%$ every year during the last decade, making it the sixth most common form of cancer in men and the fifth in women [1]. Early diagnosis is important for prognosis and survival [2]. Furthermore, nonmelanoma skin cancer (excluding basal cell carcinomas) is the second most common type of cancer for both men and women in Sweden. The number of cases per 100,000 persons has increased yearly by an average of $6.5 \%$ among women and $4.9 \%$ among men during the past decade [1].

Swedish patients seeking medical advice for skin lesions of concern most often have their first contact with health care at their local primary health care center. It has proven to be challenging for general practitioners (GPs) to diagnose skin lesions correctly and to avoid unnecessary excisions or referrals to dermatologists with the associated increase in health care costs [3].

Previous studies have shown that dermoscopy in addition to clinical examination can increase the diagnostic accuracy of pigmented skin lesions when performed by trained physicians. Results from a meta-analysis showed that physicians who underwent at least minimal training in dermoscopy increased their diagnostic accuracy in identifying melanoma when examining pigmented skin lesions [4]. A study by Rosendahl et al showed increased diagnostic accuracy when examining both melanocytic and nonmelanocytic skin lesions using dermoscopy in addition to clinical examination [5].

To increase the diagnostic accuracy, some form of training in dermoscopy seems necessary. Binder et al showed improved diagnostic accuracy among dermatologists after 9 hours of dermoscopy training, whereas the diagnostic accuracy without previous training declined [6]. Positive effects on diagnostic accuracy for both melanocytic and nonmelanocytic lesions have also been shown among GPs after dermoscopy training [7-9]. The amount of training among GPs in diagnosing skin tumors may vary between different countries [10]. In Sweden, no training in dermoscopy is required for GPs during their residency program [11].

The aim of this study was to examine the effects of a 1-day training course in dermoscopy among Swedish residents and specialists in general medicine directly after the course and after a 6-month follow-up period.

\section{Materials and Methods}

The study was designed using an intervention group of GPs that underwent a 1-day training course in dermoscopy and a control group that did not undergo any education. Approximately 6 weeks before the training course, the intervention group took an online test consisting of multiple-choice ques- tions asking to diagnose 30 dermoscopy cases. The participants were then asked to take the same test directly after the course and again after 6 months. The control group completed the same test twice with a 6-month interval between tests in order to avoid recall bias. Participants and controls were informed of the number of correct answers after each test occasion, but the correct answers to the cases were not released until after the last test occasion.

The 30 multiple-choice questions in the online test contained a clinical and a dermoscopic image of a single lesion followed by 4 different diagnostic alternatives with only 1 diagnosis being correct. The test had a time limit of 20 minutes. The cases consisted of 9 melanomas, 8 nevi, 3 basal cell carcinomas, 2 squamous cell carcinomas, 3 seborrheic keratoses, 2 dermatofibromas, 1 squamous cell carcinoma in situ, 1 solar lentigo, and 1 angioma. The pictures were taken with a smartphone (iPhone 4, Apple, Cupertino, California, USA) and a hand-held dermatoscope compatible with the smartphone (FotoFinder Handyscope, FotoFinder Systems GmbH, Bad Birnbach, Germany) at the Department of Dermatology at Sahlgrenska University Hospital in Gothenburg, Sweden. The malignant diagnoses were all confirmed by histopathological examination, whereas most of the benign cases were diagnosed by clinical examination only by an expert in dermoscopy. None of the images in the test were shown during the course.

The dermoscopy course used in the study was certified by Lipus (The Physicians' Institute for the Professional Development of Healthcare), a national organization that certifies courses for physicians in Sweden (www.lipus.se). The course comprised 5 separate lectures on the dermoscopic technique and terminology (30 minutes); an introduction to "pattern analysis" and the diagnosis of benign nonmelanocytic lesions (30 minutes), followed by 3 lectures focusing on the dermoscopic diagnosis of melanocytic lesions (60 minutes), basal cell carcinoma (30 minutes) as well as squamous cell carcinoma and precursor lesions (30 minutes). These lectures were interlaced with 2 case-based interactive sessions (45 and 60 minutes, respectively) and the courses concluded with a question-and-answers session (15 minutes). All participants were provided with PDF files of the presentations. The dermoscopy algorithm taught was "pattern analysis" [12].

An online questionnaire about previous experience concerning dermoscopy was also sent to both groups. The questionnaire contained questions regarding previous training in dermoscopy, how many patients seeking care for skin lesions of concern they met every week, whether they had access to a dermatoscope and, if so, how often they used dermoscopy during their clinical examination.

The intervention group consisted of resident physicians and specialists in general medicine that participated in the 1-day training course. The control group consisted of a ran- 
Table 1. Previous Experience With Dermoscopy Among

Physicians With Paired Observations in Both Groups

\begin{tabular}{|c|c|c|}
\hline & $\begin{array}{l}\text { Intervention Group } \\
\qquad(n=27)\end{array}$ & $\begin{array}{l}\text { Control Group } \\
\quad(n=16)\end{array}$ \\
\hline \multicolumn{3}{|l|}{ Clinical experience } \\
\hline Specialist in general medicine & $16(59.3 \%)$ & $2(12.5 \%)$ \\
\hline Resident physician in general medicine & $11(40.7 \%)$ & $14(87.5 \%)$ \\
\hline \multicolumn{3}{|c|}{ Do you have access to dermatoscope at your clinic? } \\
\hline Yes & $26(96.3 \%)$ & $14(87.5 \%)$ \\
\hline No & $1(3.7 \%)$ & $2(12.5 \%)$ \\
\hline \multicolumn{3}{|c|}{ How many patients seeking advice for skin lesions of concern do you see every week? } \\
\hline $0-3$ & $19(70.4 \%)$ & $13(81.3 \%)$ \\
\hline$\geq 4$ & $8(29.6 \%)$ & $3(18.8 \%)$ \\
\hline \multicolumn{3}{|c|}{ How often do you use dermoscopy to diagnose skin lesions? } \\
\hline Once a week or more often & $19(70.4 \%)$ & $11(68.8 \%)$ \\
\hline A few times per month or never & $8(29.6 \%)$ & $5(31.3 \%)$ \\
\hline \multicolumn{3}{|c|}{ Do you have any previous educational experience in dermoscopy? } \\
\hline Yes & $15(55.6 \%)$ & $8(50.0 \%)$ \\
\hline No & $12(44.4 \%)$ & $8(50.0 \%)$ \\
\hline
\end{tabular}

Table 2. Test Results Among the Course Participants Before vs After Course vs 6 Months After Course

\begin{tabular}{|c|c|c|c|}
\hline & Test Before Course & Test After Course & $\begin{array}{c}\text { Test } 6 \text { Months After } \\
\text { Course }\end{array}$ \\
\hline Median (interquartile), $\mathrm{n}=18$ & $14.0(4.0)$ & $20.5(3.0)$ & $20.0(5.0)$ \\
\hline P value ${ }^{a}$ before vs after course & \multicolumn{3}{|c|}{$(<0.01)$} \\
\hline P value before vs 6 months after course & \multicolumn{3}{|c|}{$(<0.01)$} \\
\hline P value a after vs 6 months after course & \multicolumn{3}{|c|}{$(0.3)$} \\
\hline
\end{tabular}

${ }^{a}$ Wilcoxon signed rank test.

domly selected mix of resident physicians and specialists in general medicine who were invited via email. The goal was to recruit the same number of participants in both groups. The collection of all data was retrieved by email invitations with links to the online test and questionnaire.

\section{Statistical Analysis}

STATA (StataCorp LLC, Texas, USA) was used for the statistical analysis. Since normal distribution could not be assumed owing to the small sample size, Wilcoxon signed rank test was used to calculate changes in individual test scores after completion of the training course (cases) and after 6 months (cases and controls). The results of the questionnaire are presented with simple descriptive statistics.

\section{Results}

The intervention group initially consisted of 56 physicians of which 41 answered the first test, 32 took the second test, and
26 also replied to the last test. This resulted in 27 cases with paired observations between the first and second test and 18 cases in which the physicians performed all 3 tests. The control group consisted of 121 physicians invited by email. Among these, 30 answered the first test and 16 also took the second test with 16 paired observations.

Table 1 presents descriptive data received from the questionnaires of the 27 participants in the intervention group that had at least 1 paired observation and the 16 participants in the control group with paired observations.

Among the 27 paired observations in the intervention group, the median number of correct answers on the first test before the course was 13 out of 30 cases (43.3\%) (interquartile range $[\mathrm{IQR}]=4.0)$. In the second test immediately after the course, the median was 21 points $(70.0 \%)(\mathrm{IQR}=$ $4.0)$, which meant an improvement of the median score by 8 points or $26.7 \%$ ( $\mathrm{P}<0.01)$.

Table 2 shows the results of the 18 participants who also took the test a third time after 6 months. A significant 
change was observed between the first and second test, but no significant change was demonstrated between the second and third test.

Among the 16 physicians in the control group with paired observations, the median score for the first test was 13.5 points $(\mathrm{IQR}=5.0)$ compared with 15.5 points $(\mathrm{IQR}=4.5$ ) in the second test after 6 months. The improvement of 2.0 points was not significant $(\mathrm{P}=0.06)$.

The baseline results (first test) were similar in both groups with 13.5 points $(\mathrm{IQR}=5.0)$ in the intervention group and 13.0 points $(\mathrm{IQR}=4.0)$ in the control group $(\mathrm{P}=0.093)$.

\section{Discussion}

After completion of the 1-day training course in dermoscopy, the course participants increased their diagnostic accuracy measured as an increase of their median test scores by 8.0 points. The improvement was statistically significant compared with the control subjects and was also considered to be a relevant improvement. At the follow-up test after 6 months, there was no significant difference in test results among participants, meaning that the newfound knowledge seemed to persist over time. Although the control group also showed a small improvement of 2 points in their test scores after 6 months, the change was not significant.

The prior educational experience in dermoscopy was similar in both groups as was the number of patients they met and how often they used dermoscopy in their clinical practice. The main difference between the groups was the distribution of specialists and resident physicians, with $59.3 \%$ being specialists in the intervention group compared with only $12.5 \%$ in the control group. Nevertheless, both groups showed similar baseline test results, which indicates that selection bias was not a major problem.

Our results are in line with those of a Dutch study by Secker et al in which 309 GPs underwent a 1-day course in dermoscopy and took a test with 20 cases before the course and 3 months after. The authors found that the diagnostic accuracy increased for all pigmented skin lesions except nevi [9]. In our study, we have not carried out subgroup analyses on the diagnostic accuracy for different lesion types considering our small sample size.

Another study by Argenziano et al also showed positive effects among GPs in Spain and Italy using dermoscopy when diagnosing skin lesions [7]. In this study, GPs underwent a 1-day educational program in skin cancer diagnostics including dermoscopy training using the "3-point checklist" algorithm [13]. Physicians were randomized into 2 groups where 1 group was allowed to use dermoscopy in addition to the clinical examination and 1 group was allowed only a clinical examination. The group using dermoscopy had a $25 \%$ higher sensitivity in detecting lesions with a suspicion of skin cancer.
The number of participants and controls was unfortunately smaller than we had first hoped for despite many reminders to those invited. However, the addition of a control group strengthened our results showing that a 1-day training course improved the test results and that there was no obvious recall bias despite repeating the same online test. The follow-up time of 6 months between the tests was also longer than that of previous studies [9].

\section{Conclusions}

Since the overall incidence of skin cancer is increasing in Sweden and GPs are often the first contact with health care that the patients encounter, good diagnostic accuracy of skin lesions of concern in primary care is essential. In previous studies, it has been shown that dermoscopy in addition to clinical examination increases the diagnostic accuracy among both dermatologists and GPs $[4,5,7,8]$. Increased competence among GPs can potentially also decrease the number of unnecessary excisions and referrals to dermatologists. The results of this study show the positive effects of a 1-day training course in dermoscopy for GPs. Hopefully, this can spread the interest in dermoscopy within primary care.

\section{References}

1. National Board of Health and Welfare. Cancer incidence in Sweden 2017 [in Swedish]. 2018. Retrieved from: https://www. socialstyrelsen.se/globalassets/sharepoint-dokument/artikelkatalog/statistik/2018-12-50.pdf. Accessed March 5, 2019.

2. Confederation of Regional Cancer Centres in Sweden. Malignant melanoma: national guideline [in Swedish]. 2018. Retrieved from: https://www.cancercentrum.se/globalassets/cancerdiagnoser/hud/ vardprogram/nationellt-vardprogram-malignt-melanom.pdf. Accessed March 5, 2019.

3. Lindelöf B, Hedblad MA, Ringborg U. Nevus or malignant melanoma? Correct diagnostic competence results in lower costs [in Swedish]. Läkartidningen. 2008;105(39):2666-2669.

4. Vestergaard ME, Macaskill P, Holt PE, Menzies SW. Dermoscopy compared with naked eye examination for the diagnosis of primary melanoma: a meta-analysis of studies performed in a clinical setting. Br J Dermatol. 2008;159(3):669-676.

5. Rosendahl C, Tschandl P, Cameron A, Kittler H. Diagnostic accuracy of dermatoscopy for melanocytic and nonmelanocytic pigmented lesions. J Am Acad Dermatol. 2011;64(6):1068-1073.

6. Binder M, Puespoeck-Schwarz M, Steiner A, et al. Epiluminescence microscopy of small pigmented skin lesions: Short-term formal training improves the diagnostic performance of dermatologists. J Am Acad Dermatol. 1997;36(2):197-202.

7. Argenziano G, Puig S, Zalaudek I, et al. Dermoscopy improves accuracy of primary care physicians to triage lesions suggestive of skin cancer. J Clin Oncol. 2006;24(12):1877-1882.

8. Rosendahl C, Williams G, Eley D, et al. The impact of subspecialization and dermatoscopy use on accuracy of melanoma diagnosis among primary care doctors in Australia. J Am Acad Dermatol. 2012;67(5):846-852. 
9. Secker LJ, Buis PA, Bergman W, Kukutsch NA. Effect of a dermoscopy training course on the accuracy of primary care physicians in diagnosing pigmented lesions. Acta Derm Venereol. 2017;97(2):263-265.

10. Cate OT. Medical education in the Netherlands. Med Teach. 2007;29(8):752-757.

11. National Board of Health and Welfare. Regulations and general guidelines for doctor's specialist medical training (SOSFS 2015:8) [in Swedish]. Acting General Counsel Pär Ödman; 2015. Re- trieved from: http://www.socialstyrelsen.se/Lists/Artikelkatalog/ Attachments/19743/2015-3-1.pdf. Accessed April 26, 2017.

12. Marghoob AA, Braun R. Proposal for a revised 2-step algorithm for the classification of lesions of the skin using dermoscopy. Arch Dermatol. 2010;146(4):426-428.

13. Soyer HP, Argenziano G, Zalaudek I, et al. Three-point checklist of dermoscopy: a new screening method for early detection of melanoma. Dermatology. 2004;208(1):27-31. 